Classification

Physics Abstracts

$63.50-76.30-61.40$

\title{
Relaxation rate distribution and decay profile : two fracton relaxation
}

\author{
S. Alexander $\left({ }^{*}\right)$, O. Entin-Wohlman $\left({ }^{* *}\right)$ and R. Orbach \\ Department of Physics, University of California, Los Angeles, CA 90024, U.S.A.
}

(Reçu le 4 février 1985, accepté sous forme définitive le 23 avril 1985)

\begin{abstract}
Résumé. - La distribution de probabilité pour le taux de relaxation à deux fractons, $1 / T_{1}$, d'un réseau de spins est calculée pour un réseau fractal. Le temps de retour à l'équilibre est gouverné initialement par une exponentielle étendue, puis un crossover à une dépendance plus lente qu'une exponentielle, mais plus rapide que toute loi de puissance, se produit. La moyenne, $1 / T_{1}^{\text {ave }}$, est calculée et comparée à des estimations antérieures.

Abstract. - The probability distribution is calculated for the largest two fracton spin lattice relaxation rate, $1 / T_{1}$, in a fractal network. The initial long-time return to equilibrium is governed by a stretched exponential time dependence, afterwards a crossover to a time dependence slower than exponential but faster than any power law is obtained. The average, $1 / T_{1}^{\text {ave }}$, is calculated and compared with previous estimates.
\end{abstract}

We have calculated the one fracton relaxation process for a localized electronic impurity in a fractal network in a previous paper [1]. We applied the results to the nonradiative lifetime of excited localized electronic levels as found, as for example, in fluorescent line narrowing experiments. The calculation of the spin lattice relaxation time for a paramagnetic (or nuclear) moment in a fractal network involves relatively much smaller changes in electronic energy. The energies involved are so low that the relevant vibrational excitations are expected to be extended phonons and not fractons for single vibrational quanta relaxation [2]. As a consequence, one would expect processes involving the inelastic scattering of vibrational quanta to dominate at higher temperatures. These quanta can be phonons at relatively low temperatures $\left(k_{\mathrm{B}} T / \hbar\right.$ below the crossover frequency $\left.\omega_{\mathrm{c}}\right)$, and fractons at relatively higher temperatures $\left(k_{\mathrm{B}} T / \hbar\right.$ above the crossover frequency $\left.\omega_{\mathrm{c}}\right)$.

The purpose of this paper is to calculate the two fracton contribution to the spin lattice relaxation rate, $1 / T_{1}$, for a paramagnetic (or nuclear) moment in a fractal network. We shall find that the time dependence of electronic level occupation will cross over from a stretched exponential to a dependence similar to that found in reference [1] slower than exponential (or stretched exponential) but faster than any power law as time progresses. When rapid cross-relaxation is

$\left(^{*}\right)$ Permanent address : Racah Institute, The Hebrew University, Jerusalem, Israel.

(**) Permanent address : School of Physics and Astronomy, Tel Aviv University, Tel Aviv, Israel 69978. 
present, the decay is exponential and is governed by the average relaxation rate. The temperature dependence one obtains is sensitive to the details of the model used. For this reason, we find a somewhat different relationship to the fractal dimensions from that obtained by Stapleton et al. [3] with a slightly different model. We believe this calculation should be relevant to all electronic and nuclear $T_{1}$ measurements in glasses, amorphous materials, polymers, and organic molecules of large molecular weight.

We calculate the spin lattice relaxation rate due to the inelastic scattering of two fractons of an electronic or nuclear magnetic impurity in a fractal network. The fractons are localized lattice vibrations [4] at distances $L$ and $L^{\prime}$ from the magnetic impurity, and are of energy $\omega_{\alpha}$ and $\omega_{\alpha}+\omega_{0}$, where $\omega_{0}$ is the Zeeman energy and $\alpha$ represents the fracton mode index. The relaxation rate at different impurity sites is then different because of the fluctuations in the availability of correct energy vibrational states near the impurity. We deal with the statistics of finding localized vibrations of appropriate energy and position by calculating the largest relaxation rate probability at a given site from the $a$ priori probability for a given relaxation rate.

In order to calculate the two fracton relaxation rate, we make use of the form for the (localized) fracton wave function which we introduced in [1]. We take the electron-fracton interaction to be proportional to the gradient of that wave function. Using the usual Bose factors for absorption and emission of quantized fractons, we find a rate proportional to (the general form for the relaxation rate can be found in Ref. [5]),

$$
\begin{aligned}
W\left(\omega_{\alpha}, L, L^{\prime}\right) \propto\left(l_{\omega_{\alpha}}\right)^{-2 d} \omega_{\alpha}^{4 q-2}\left[1 / \delta\left(L, L^{\prime}\right)\right] \times \exp [ & \left.-\left(L / l_{\omega_{\alpha}}\right)^{d_{\min }}-\left(L^{\prime} / l_{\omega_{\alpha}}\right)^{d_{\min }}\right] \\
& \times\left\{\left[\exp \left(\beta \omega_{\alpha}\right)\right] /\left[\exp \left(\beta \omega_{\alpha}\right)-1\right]^{2}\right\}\left(\omega_{\alpha}^{2} / \Delta^{4}\right) .
\end{aligned}
$$

Here, we have neglected $\omega_{0}$ compared to $\omega_{\alpha}$. In equation (1), the factor $l_{\omega_{\alpha}}^{-2 \bar{d}}$ arises from the normalization of the localized fracton vibrations, $\omega_{\alpha}^{4 q}$, from their spatial derivatives (here, $\left.q=\overline{\bar{d}} d_{\min } / \bar{d}\right), \omega_{\alpha}^{-2}$ from the normal mode expansion, and $\delta\left(L, L^{\prime}\right)$ represents the combined widths of the electronic and fracton states. In these definitions, $l_{\omega_{\alpha}}$ is the characteristic length scale of the fracton modes. It is the only length scale in the problem [2], and we take it equal to the fracton localization length. The dimensionalities are $: \bar{d}$, the fractal (Hausdorff) dimensionality; $d_{\min }$ an index which relates the Euclidean length $L$ to the "chemical length $l$, with $d_{\min }$ defined in reference [6]; and $\overline{\bar{d}}$ the fracton dimensionality [7]. The expression above is written for the relaxation rate of a Kramers doublet [5], with $\Delta$ representing the splitting between the ground and excited doublets. For non-Kramers transitions, we replace the factor $\omega_{\alpha} / \Delta^{2}$ by $1 / \Delta$ in (1). A more detailed discussion of the details of equation (1) will be given elsewhere [8]. We re-write (1) as

$$
W\left(\omega_{\alpha}, L, L^{\prime}\right)=W_{\mathrm{m}}\left(\omega_{\alpha}\right)\left[\delta(0,0) / \delta\left(L, L^{\prime}\right)\right] \times \exp \left[-\left(L / l_{\omega_{\alpha}}\right)^{d_{\min }}-\left(L^{\prime} / l_{\omega_{\alpha}}\right)^{d_{\min }}\right],
$$

where

$$
W_{\mathrm{m}}(z)=W_{1} z^{a}\left\{\exp (z) /[\exp (z)-1]^{2}\right\}, \quad z=\beta \omega_{\alpha},
$$

and $a=4 q+2 \overline{\bar{d}}, 4 q+2 \overline{\bar{d}}-2$ for Kramers, non-Kramers transitions, respectively. In equation (3), $W_{1}=W_{0} \beta^{-a}, \beta=1 / k_{\mathrm{B}} T$, and $W_{0}$ is a constant divided by $\delta(0,0)$. When $a>2$, the function $W_{\mathrm{m}}(z)$ is peaked at $z_{\max }\left(z_{\max }=0\right.$ for $\left.a=2\right)$, and its maximal value is of the order $W_{1}$. We denote this maximum available relaxation rate at a given temperature by $W_{\max }$

We next consider the probability density $\mathfrak{S}(W)$ for a given relaxation rate $W$. Three considerations contribute to $\mathfrak{T}(W):$ (i) the probability of finding a fracton state in the spatial range $L$, $L+\mathrm{d} L$ with energy between $\omega_{\alpha}, \omega_{\alpha}+\mathrm{d} \omega_{\alpha}$. This is given by $\bar{d} L^{\bar{d}-1} \mathrm{~d} L N\left(\omega_{\alpha}\right) \mathrm{d} \omega_{\alpha}$, where $N\left(\omega_{\alpha}\right)$ is the fracton energy density of states; (ii) the probability to find a second fracton state in the 
range $L^{\prime}, L^{\prime}+\mathrm{d} L^{\prime}$ with energy between $\omega_{\alpha}, \omega_{x}+\delta$. This is given by $\bar{d}\left(L^{\prime}\right)^{\bar{d}-1} \mathrm{~d} L^{\prime} N\left(\omega_{\alpha}\right) \delta\left(L, L^{\prime}\right)$; (iii) a $\delta$-function imposing $W\left(\omega_{\alpha}, L, L^{\prime}\right)=W$. Combining and integrating

$$
\mathfrak{T}(W)=\int \mathrm{d} \omega_{\alpha} \mathrm{d} L \mathrm{~d} L^{\prime}(\bar{d})^{2}(L)^{\bar{d}-1}\left(L^{\prime}\right)^{\bar{d}-1} \delta\left(L, L^{\prime}\right) \times N^{2}\left(\omega_{\alpha}\right) \delta\left[W\left(\omega_{\alpha}, L, L^{\prime}\right)-W\right] .
$$

We now use our expression for $\mathfrak{T}(W)$ to write the probability density $P(W)$ for the largest relaxation rate seen at a given impurity site. We have,

$$
P(W)=\mathfrak{T}(W) \exp [-F(W)], \quad \text { with } \quad F(W)=\int_{W}^{W_{\max }} \mathrm{d} W_{1} \mathfrak{T}\left(W_{1}\right) .
$$

The exponential insures that no relaxation rate is larger than $W$. The largest relaxation rate probability will be used below to find the dominant features of the spin-lattice relaxation rate $1 / T_{1}$.

As we have noted elsewhere [1], the origin of the level width $\delta$ needs to be prescribed. If the fractons are perfectly sharp in energy, then $\delta$ is just the self-consistent width $W$. This requires in the two fracton case a very complex analysis which we reserve to a subsequent publication [8]. Here, for simplicity, we take $\delta\left(L, L^{\prime}\right)=\delta$, a constant independent of position and temperature.

It is more convenient to calculate $F(W)$ directly. Inserting equation (4) into (5), we find

$$
F(W)=\eta B(\eta, \eta+1)(\overline{\bar{d}})^{2} \beta \delta \int_{z_{1}}^{z_{2}}\left(\mathrm{~d} z / z^{2}\right)\left[\ln \left(W_{1} / W\right)+\ln f(z)\right]^{2 \eta},
$$

where $\eta=\bar{d} / d_{1}, f(z)=z^{a} \exp (z) /[\exp (z)-1]^{2}$, and $B(\eta, \zeta)$ is the Beta function (in our case, simply a numerical constant). Here we have taken $l_{\omega_{\alpha}}^{\bar{d}} \sim \omega_{\alpha}^{-\bar{d}}$, so that $N\left(\omega_{\alpha}\right) l_{\omega_{\alpha}}^{\bar{d}}=\overline{\bar{d}} / \omega_{\alpha}[1]$.

The integration limits in equation (6) are rather complicated. Investigation of equations (4) and (5) reveals that they must be chosen so that the expression in the square brackets of (6) is positive. Limiting ourselves to the case $a>2$ (this will be shown at the end of the paper to be the case most often encountered) in which $f(z)$ is double valued, one sees that $z_{1}, z_{2}$ are the solutions of the equation $W_{1} f(z)=W[9]$.

We consider the small $W / W_{1}$ limit of equation (6) (note that $W_{1}$ is of the order of $W_{\text {max }}$ ) and temperatures higher than the lowest cutoff frequency [9], $\omega_{\mathrm{c}}$, such that $\beta \omega_{\mathrm{c}} \ll 1$. Because $f(z)$ is a double-valued function, there are two contributions for $F(W)$ : the small and large $z$ regions, respectively. The large $z$ region of the integration in (6) contributes a term of the order $\beta \delta\left[\ln \left(W_{1} / W\right)\right]^{2 \eta}$. The contribution of the small $z$ region depends upon the lower bound of the integration in (6) $\left(z_{1}\right.$ or $\beta \omega_{\mathrm{c}}$, respectively [9]) and consequently upon $W_{\mathrm{c}}=W_{1}\left(\beta \omega_{\mathrm{c}}\right)^{a-2}<W_{1}$. We find

$$
\left.F(W)\right|_{\substack{\text { region } z \\ \text { region }}} \propto \begin{cases}\alpha_{1}\left(\delta / \omega_{\mathrm{c}}\right)\left[\ln \left(W_{\mathrm{c}} / W\right)\right]^{2 \eta}, & W<W_{\mathrm{c}} \\ \alpha_{1}(\beta \delta)\left[W_{1} / W\right]^{1 /(a-2)}, & W>W_{\mathrm{c}}\end{cases}
$$

where $\alpha_{1}$ is a numerical constant. The contribution (7a) dominates the contribution of the large $z$ region because $\beta \omega_{\mathrm{c}} \ll 1$. Thus, the limiting behaviour of $F(W)$ is dominated by the small $z$ region, i.e., by small frequencies. This is peculiar to Raman scattering of fractons (as contrasted to phonons), and is a consequence of the frequency dependence of the localization length. It affects the distribution of $W$ at small $W$, but not the average relaxation rate.

The return to equilibrium is given by the Laplace transform of (5) :

$$
P(t)=\int_{0}^{W_{\max }} \mathrm{d} W[\exp (-W t)] P(W) .
$$


At long times, the $W$ integration is governed by the small $W$. Inserting equation (7) into (8), and using the saddle-point method of integration, we find, to leading order, that the contribution of $W<W_{\mathrm{c}}$ is

$$
P(t)_{W<W_{c}} \propto\left(\delta / \omega_{c}\right)\left(\ln \tau_{1}\right)^{\eta-1 / 2}\left(\tau_{1}\right)^{-\alpha_{2}\left(\delta / \omega_{c}\right)\left(\ln \tau_{1}\right)^{2 \eta-1}}
$$

with the reduced time variable $\tau_{1}=(1 / 2 \eta)\left(\omega_{\mathrm{c}} / \alpha_{2} \delta\right) W_{\mathrm{c}} t$. Similarly, for $W>W_{\mathrm{c}}$,

$$
P(T)_{W>W_{c}} \propto\left(\tau_{2}\right)^{1 / 2(a-1)} \exp \left[-\alpha_{3}\left(\tau_{2}\right)^{1 /(a-1)}\right],
$$

with the reduced time variable $\tau_{2}=(\beta \delta)^{a-2} W_{1} t$. Above, $\alpha_{2}$ and $\alpha_{3}$ are numerical constants. We see that the long time behaviour of $P(t)$ begins as a stretched exponential (Eq. (9b)) with a time constant proportional to $(\delta)^{1-a}(\beta \delta)^{2}$. At longer times, the decay is given by equation $(9 \mathrm{a})$, and is therefore slower than exponential but faster than any power law. The time constant is proportional to $\left(\omega_{\mathrm{c}}\right)^{1-a}(\beta \delta)^{2}$. The crossover time between the two time regimes is proportional to $(\beta \delta)^{2}\left(\omega_{\mathrm{c}}\right)^{1-a}$.

As the temperature is lowered, such that $\beta \omega_{c}$ increases, the small $z$ region of the integral in (6) gradually diminishes in importance. Consequently $F(W)$ in the small $W / W_{1}$ limit becomes proportional to $\beta \delta\left[\ln \left(W_{1} / W\right)\right]^{2 \eta}$ to leading order, and the time profile becomes entirely that of (9a) with $\omega_{\mathrm{c}}$ and $W_{\mathrm{c}}$ replaced by $1 / \beta$ and $W_{1}$, respectively.

The above discussion is relevant for dilute, weakly interacting moments. When the reverse is true, and cross relaxation dominates, the return to equilibrium is expected to be exponential and governed by an average relaxation time, $1 / T \cdot{ }_{1}^{\text {ave }}$. This quantity can be calculated in two ways : (i) the first moment of $P(W)$, or (ii) directly using the " Golden Rule ». Both give the same answer which we display without proof.

We write the average relaxation rate in the form comparable to that used by Stapleton et al. [3],

$$
1 / T_{1}^{\text {ave }} \propto \int_{\omega_{c}}^{\Omega} \mathrm{d} \omega_{\alpha}\left[N\left(\omega_{\alpha}\right)\right]^{2} \omega_{\alpha}^{\bar{a}}\left\{[\exp (\beta \omega)] /[\exp (\beta \omega)-1]^{2}\right\}
$$

where $\bar{a}=a-2 \overline{\bar{d}}$. When $2<a<3$, the integral is dominated by the lower cutoff $\left(\omega_{\mathrm{c}}\right)$ and

$$
1 / T_{1}^{\text {ave }} \propto \omega_{\mathrm{c}}^{a-3} \beta^{-2} ; \quad 2<a<3 .
$$

However, for $a>3$,

$$
1 / T_{1}^{\text {ave }} \propto \beta^{1-a}
$$

Most reasonable values for the fractal exponents result in $a>3$ so that equation (11b) is more relevant. Stapleton et al. [3] assume that $q=1$ (whence $a=4+2 \overline{\bar{d}}, 2 \pm 2 \overline{\bar{d}}$ for Kramers, non-Kramers transitions, respectively). This differs from our ansatz $q=\overline{\bar{d}} d_{\min } / \bar{d}$. From (10),

$$
1 / T_{1}^{\text {ave }} \propto T^{2 \overline{\bar{d}}\left[1+2\left(d_{\min } / \bar{d}\right)\right]-1},
$$

for Kramers transitions, and

$$
1 / T_{1}^{\text {ave }} \propto T^{2 \overline{\bar{d}}\left[1+2\left(d_{\min } / \bar{d}\right)\right]-3},
$$

for non-Kramers transitions, respectively. Our ansatz for $q$ seems more natural than that of Stapleton et al. [3] in the context of a scaling description. One can, however, also have physical conditions where $q=1$ would be correct (as in Ref. [3]). Because $\bar{d}, \bar{d}$, and $d_{\min }$ are not known independently, there is at present no way of determining $q$ for the systems investigated by Stapleton et al. [3]. 
Table I. - The analytic forms for the temperature dependence of the Raman spin-lattice relaxation rate for : fractons (this paper); fractons, but with neglect of localization [3]; and localized (or extended) phonons [5], $T \ll \theta_{\mathrm{D}}, \Omega_{\mathrm{fD}}$.

Temperature dependence using percolation

Character of

Transition

Kramers

Non-Kramers

Kramers

Non-Kramers

Kramers

Non-Kramers thermal excitation

Fractons (this paper)

Fractons (this paper)

Fractons, but without localization [3]

Fractons, but without localization [3]

Localized (or extended phonons [5]

Localized (or extended phonons) [5]
Temperature dependence

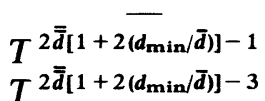

$T^{2 \overline{\bar{d}}\left[1+2\left(d_{\min } / \bar{d}\right)\right]-3}$

$T^{2 \overline{\bar{d}}+3}$

$T^{2 \overline{\bar{d}}+1}$

$T^{2 d+3}$

$T^{2 d+1}$ exponents for $d=3$

$$
\begin{gathered}
(\overline{\bar{d}}=4 / 3[7] ; \bar{d}=2.5[10] ; \\
\left.\bar{d} / d_{\min }=1.8[6]\right)
\end{gathered}
$$

$T^{4.63}$

$T^{2.63}$

$T^{5.66}$

$T^{3.66}$

$T^{9}$

$T^{7}$

To make the differences between his approach and our own more explicit, we exhibit in table I three different forms for the temperature dependence of the Raman relaxation rate caused by : fractons (present paper); fractons, but with neglect of the effects of localization [3], and localized (or extended) state phonons [5]. In addition, we evaluate the respective powers of temperature using percolation exponents. The table shows that one can obtain a broad range of temperature exponents for Raman spin-lattice relaxation on a fractal lattice.

In summary, these results (especially Eqs. (9) and (12), and Table I) exhibit the profound differences one expects for the spin-lattice relaxation process in a fractal network. The decay envelope is non-exponential (Eqs. (9)), and the temperature dependence of the average relaxation rate depends upon the fractal dimensions in a manner which can be quite complex (e.g., Eqs. (11) and (12), and Table I). It would be interesting to re-examine the explicit time decay of the magnetization in circumstances where cross relaxation is not expected (e.g., in dilute systems).

\section{Acknowledgments.}

This research has been supported by the National Science Foundation through grants DMR 81-15542, DMR 84-12898, and INT 83-12985.

\section{References}

[1] Alexander, S., Entin-Wohlman, O. and Orbach, R., J. Physique Lett. 46 (1985) L-549.

[2] Alexander, S., Laermans, C., Orbach, R. and Rosenberg, H. M., Phys. Rev. B 28 (1983) 4615 ; AleXander, S., Ann. Israel Phys. Soc. 5 (1983) 144.

[3] Stapleton, H. J., Allen, J. P., Flynn, C. P., Stinson, D. G. and Kurtz, S. R., Phys. Rev. Lett. 45 (1980) 1456 ;

Allen, J. P., Colvin, J. T., Stinson, D. G., Flynn, C. P. and Stapleton, H. J., Biophys. J. 38 (1982) 299. 
[4] Rammal, R. and Toulouse, G., J. Physique Lett. 44 (1983) L-13.

[5] Orbach, R. and Stapleton, H. J., Electronic Paramagnetic Resonance, ed. by S. Geschwind (Plenum Press, New York) 1972, p. 121.

[6] Middlemiss, K. M., Whittington, S. G. and Gaunt, D. S., J. Phys. A 13 (1980) 1835 ;

Pike, R. and Stanley, H. E., J. Phys. $A 14$ (1981) L169;

Hong, D. C. and Stanley, H. E., J. Phys. A 16 (1983) L475;

Hong, D. C. and Stanley, H. E., J. Phys. A 16 (1983) L525;

Havlin, S. and Nossal, R., J. Phys. A 17 (1984) L427;

Vannimenus, J., Nodal, J. P. and Martin, C., J. Phys. A 17 (1984) L351 ;

Rammal, R., J. Phys. A 17 (1984) L491;

Havlin, S., Djordjevic, Z. V., Majid, I., Stanley, H. E. and Weiss, G. H., Phys. Rev. Lett. 53 (1984) 178 ;

Hermann, H. J., Hong, D. C. and Stanley, H. E., J. Phys. $A 17$ (1984) L261.

[7] Alexander, S. and Orbach, R., J. Physique Lett. 43 (1982) L-625.

[8] Entin-Wohlman, O., AleXander, S. and Orbach, R., unpublished.

[9] Provided that $z_{2}<\beta \Omega$, where $\Omega$ is the fracton Debye frequency and $z_{1}>\beta \omega_{\mathrm{c}}$, where $\omega_{\mathrm{c}}$ is the lower frequency cutoff for the fracton spectrum. When $\beta \Omega<z_{2}$ or $z_{1}<\beta \omega_{\mathrm{c}}, z_{2}$ is replaced by $\beta \Omega$ and $z_{1}$ by $\beta \omega_{\mathrm{c}}$, respectively.

[10] Kapitulnik, A. and Deutscher, G., Phys. Rev. Lett. 49 (1982) 1444 ;

Voss, R. F., Laibowitz, R. B. and Allessandrini, E. I., Phys. Rev. Lett. 49 (1982) 1441. 